\title{
INTERAÇÃO ENTRE PROCESSOS DE COLONIZAÇÃO E SUCESSÃO ECOLÓGICA DE COMUNIDADES BIOINCRUSTANTES NA PRAIA DE PIEDADE.
}

Recebido em: 20/03/2006

Aceito em: 07/04/2006

\author{
Ludimila Raupp de ALMEIDA ${ }^{1}$ \\ Mariana Alves de GUIMARAENS ${ }^{1}$ \\ Betty Rose de Araújo LUZ ${ }^{1}$ \\ Fernando de Figueiredo PORTO NETO².
}

\section{RESUMO}

O presente estudo tem por objetivo avaliar as interações entre processos de colonização e sucessão ecológica de comunidades bioincrustantes dos recifes da Praia de Piedade na cidade de Jaboatão dos Guararapes, no estado de Pernambuco. Pretende também, avaliar a recuperação da comunidade de animais invertebrados após uma perturbação artificial e sua relação com os caracteres ambientais qualitativos deste ambiente, como o posicionamento relativo das diferentes zonas do recife. Neste trabalho realizamos perturbações artificiais e fomos acompanhando durante 6 meses, para avaliar o poder de regeneração do meio e comportamento das populações diante dos impactos sofridos. As amostragens da comunidade com e sem perturbação foram feitas pela contagem de organismos utilizando quadrados de $10 \times 10 \mathrm{~cm}$. A composição de espécies que recrutaram não diferiu, nas diferentes faixas amostradas. Algumas diferenças estatísticas significativas foram observadas nos quadrados de recrutamento de Cirripedia entre as datas de coleta mostrando a evolução da comunidade, porém a recuperação ao nível de abundância, pré-impacto não foi observada no período do experimento. $O$ estudo do recrutamento de invertebrados motivou a construção de um modelo com o software Stella ${ }^{\mathrm{TM}}$ que assume o recrutamento como função de entrada de novos indivíduos da população a cada passo de tempo e simula bem em ordem de grandeza o quantitativo dos invertebrados encontrados nos quadrados amostrados no campo.

Palavras chaves: bioincrustação, invertebrados marinhos, sucessão ecológica.

\section{INTERACTION PROCESSES BETWEEN COLONIZATION AND ECOLOGICAL SUCCESSION OF BIOFOULING COMMUNITIES AT PIEDADE BEACH.}

\section{ABSTRACT}

The present study has the purpose of evaluating the interactions between the colonization processes and ecological succession of biofouling communities, of reefs from Piedade Beach at Jaboatão dos Guararapes City, Pernambuco State. We also intend to evaluate the recovery of the invertebrate animal community after an artfitial perturbation and their relation with the qualitative environmental characteristics of this environment, with the positioning on the different reef zones. In this work we manipulated the reefs communities and followed the open patches for about 6 months to evaluate the regeneration processes of the media, and their behavior in front of the different manipulation impacts. The community sampling with and without perturbation were made by the counting of organisms using $10 \times 10 \mathrm{~cm}$ quadracts. The species composition of recruiting organisms did not differ, on the different sampled levels. Some significant statistical differences were observed on the sampling recruitment of Cirripedea between the sampling data showing the community evolution in time. However the recovery of the preimpact level were not observed during the experiment time. The recruitment study of invertebrates motivated the building of a model with the software Stella ${ }^{\mathrm{TM}}$ that assumes the recruitment as a function of new individuals entering the population at each time step and

Contatos: ${ }^{1}$ Universidade de Pernambuco, Instituto de Ciências Biológicas, Departamento de Biologia, Rua Arnóbio Marques 310, Santo Amaro, Recife - PE, 50100-130, Brazil.

Fax/Phone: (21) 2285-3471

E - mail: mguimaraens@hotmail.com

2Departamento de Oceanografia da UFPE. 
ALMEIDA, et al. Interação entre processos de colonização e sucessão interação entre processos de colonização e sucessão ecológica de comunidades bioincrustantes na praia de Piedade.

simulates well the general quantitative values of the invertebrate community found on the sampled quadracts in the field.

Keywords: biofouling, marine invertebrates, ecological succession.

\section{INTRODUÇÃO}

A sucessão ecológica é um processo natural que ocorre através das mudanças que se verificam nos ecossistemas após a destruição parcial da comunidade, que ocorrem ao longo do tempo, como conseqüência da colonização do substrato, e das interações biológicas que são importantes fatores estruturadores de comunidades (SAUER-MACHADO et al., 1992).

Ela resulta da modificação do ambiente físico pela comunidade e de interações de competição e coexistência ao nível de população. A sucessão é controlada pela comunidade, muito embora o ambiente físico determine o padrão e a velocidade da mudança, muitas vezes limitando também a extensão do desenvolvimento (ODUM, 1988), estas mudanças são muito evidentes quando há um distúrbio externo, perturbação por humanos (PINTOCOELHO, 2000). Distúrbios bióticos e abióticos podem alterar a estrutura da comunidade marinha alterando padrões de sucessão ecológica formando um conjunto de manchas que em conjunto com o recrutamento aleatório podem formar comunidades com características de mosaicos (SAUER-MACHADO et al., 1992). Existem estudos que encontram uma correlação ente a estrutura e dinâmica populacional de Chthamalus (crustáceo) e a disponibilidade de larvas e sua relação com fatores oceanográficos como a ressurgência (SKINNER, 2003).

Características físicas ou as variáveis biológicas tais como a competição e a predação explicam geralmente diferenças espaciais e mudanças temporais nas faixas dos recifes, que são dominadas por diferentes espécies. A presença de determinadas espécies, sua abundância relativa, qualidade e quantidade de larvas plâncton são fatores que influenciam na estrutura das comunidades de Cirripedia e Brachidontes (APOLINÁRIO, 1999).

A dinâmica da sucessão em comunidades pode ainda ser influenciada por interações entre o recrutamento de espécies e por características das aberturas criadas por distúrbios, tais como relação do perímetro e da área. As aberturas da região entre-marés estabelecidas como conseqüência de distúrbios ambientais, oferecem o substrato para o estabelecimento de novas espécies. Estas aberturas criadas dentro das comunidades sésseis em substrato emergente podem ser colonizadas pelas larvas e propágulos que vêm da coluna de água, e pela migração lateral da espécie vizinha, incluindo o crescimento vegetativo, de macroalgas e espécies coloniais (TANAKA \& MAGALHÃES, 2002).

O litoral de Pernambuco, com aproximadamente $187 \mathrm{~km}$ de extensão, caracteriza-se pela presença de recifes de arenito. Esses recifes são afloramentos marinhos que formam cordões paralelos à costa, sendo diques naturais, que nem sempre estão emersos durante a baixa-mar. Resultam da consolidação ou litificação da areia da praia por cimentação calcárea ou ferruginosa, processos esses relacionados com a tectônica do fundo marinho e a subseqüente diminuição do nível da água do mar; são constituídos essencialmente por quartzo e bioclastos, principalmente de moluscos e algas (SOUSA \& COCENTINO, 2004). Muitos destes recifes constituem o principal substrato duro para fixação de algas bentônicas, como também de uma fauna associada bastante diversificada, sendo então um ecossistema com fortes interações ecológicas.

As comunidades epibênticas marinhas, localizadas nos corais, possuem uma taxa de renovação de espécies alta, as modificações ocorrem em intervalos de tempo relativamente curtos, de poucos meses a alguns anos, muitos dos organismos integrantes são sésseis o que os tornam, animais de grande potencial para o estudo da ecologia pura (CHASILEW, 1998). Há várias linhas de pensamento a respeito do rumo da sucessão ecológica em organismos bioincrustantes, falam alguns em um processo ordenado, onde as espécies tardias dependem das iniciais, e outros que questionam 0 andamento do processo, afirmando que ele é imprevisível (LUBCHENCO \& MENGE, 1978). Fatores abióticos e bióticos influenciam o crescimento de animais incrustados em superfícies como recifes, e são de grande importância para a determinação das comunidades bentônicas no ambiente marinho (COUTINHO, 2002).

Nas últimas décadas, a questão da distribuição espacial das espécies, da ocupação dos 
substratos e da dinâmica populacional, vem recebendo muita atenção dos ecólogos, e foi desenvolvido um novo ramo científico denominado supply side ecology (LEWIN, 1986), que considera a importância do assentamento e recrutamento como fatores biológicos estruturadores de comunidades (COUTINHO, 2002). Dentre os invertebrados as comunidades sésseis são as mais estudadas, seja pela relativa facilidade de acesso e manipulação quanto pela facilidade de serem transportadas para laboratório (SKINNER, 2003).

Ao longo de recifes de arenito, ou recifes coralinos, poças de maré são formadas, representando então uma pequena biota intertidal, onde o plâncton tem importante papel na teia trófica e dispersão de larvas (KIM \& DeWREDEE, 1996). Poças de maré também são importantes áreas para a dinâmica de muitas populações de micro-crustáceos, como exemplo Copepoda Harpacticoida (JOHNSON, 2001) e Copepoda parasitas (GROBLER et. al., 2002).

As regiões costeiras e seus recifes associados são susceptíveis a impactos antropogênicos, devido à grande concentração populacional e as atividades econômicas, incluindo os complexos portuários, o turismo e o lazer. Esses fatores podem causar mudanças nas comunidades de algas e fauna associada, que são sensíveis às modificações introduzidas pelo homem (SOUSA \& COCENTINO, 2004). Na Praia de Piedade existe uma alta densidade populacional e presença de muitos hotéis, recebendo um grande aporte de moradores, comerciantes e turistas que contribuem com a degradação do ecossistema, sendo importante avaliar a condição desta área, caracterizada por faixa recifal próxima à praia.

O nosso objetivo geral foi quantificar e identificar os organismos pertencentes à comunidade de invertebrados da zona superior dos recifes de arenito da Praia de Piedade, a fim de avaliar o processo de sucessão ecológica após perturbação artificial. O objetivo específico foi avaliar o processo de colonização do substrato impactado artificialmente (raspagens) pela quantificação dos recrutas, e a evolução temporal da comunidade de invertebrados dominada por Cirripedia e Brachidontes. Desta forma este trabalho contribui com uma avaliação de parte da dinâmica relacionada com o recrutamento e a possibilidade da manutenção da biodiversidade através de processos de colonização de substratos de recifes costeiros, que podem acarretar na recuperação da comunidade.

\section{DESCRIÇÃO DA ÁREA}

O local de realização deste trabalho situa-se na Praia de Piedade - Jaboatão dos Guararapes, localizado ao Sul da região metropolitana do Recife, entre os paralelos $08^{\circ} 09^{\prime} 17^{\prime}$ e $08^{\circ} 11^{\prime} 19^{\prime}$ ' de latitude sul, possuindo uma extensão de $5,6 \mathrm{~km}$. Com clima Tropical Atlântico, temperatura média do ar próxima $26^{\circ} \mathrm{C} \mathrm{com}$ variação média anual de $2,7^{\circ} \mathrm{C}$. Precipitação média anual em torno de $1720 \mathrm{~mm}$, em meses de setembro e fevereiro abaixo de $100 \mathrm{~mm}$ (período seco) e entre meses de março e agosto acima de $100 \mathrm{~mm}$ (período chuvoso) (CAVALCANTI \& KEMPF, 1969).

A Praia de Piedade possui uma boa infra-estrutura hoteleira, prédios habitacionais, bares e restaurantes, o que o torna um local bastante freqüentado e com grande fluxo turístico. Muitas pessoas concentram-se sobre os recifes para caminhar, tomar sol, estacionar bicicletas e pescar, inclusive quebrando-o para obtenção de iscas.

Em substratos consolidados na zona litorânea verifica-se uma tendência de distribuição dos organismos em faixas horizontais. No local estudado verificou-se a ocorrência bem delimitada de 3 áreas com predominância de Brachidontes, Cirripedia e em seguida de algas, na direção praia-mar. Para o estudo foram amostradas 2 estações, respectivamente nas zonas de Brachidontes e de Cirripedia.

A estação 1 denominada Brachidontes localizada na região mais próxima da praia, caracteriza-se por apresentar uma superfície mais baixa, sendo uma área mais pisoteada pelos banhistas, apresentando poças de maré. A estação 2 denominada Cirripedia está situada em área mais elevada do recife, sendo mais exposta aos raios solares, porém mais próximo da zona de arrebentação. 
ALMEIDA, et al. Interação entre processos de colonização e sucessão interação entre processos de colonização e sucessão ecológica de comunidades bioincrustantes na praia de Piedade.

\section{MATERIAL E MÉTODOS}

Neste trabalho foram realizadas perturbações artificiais através da raspagem do substrato, utilizando-se como medida padrão, quadrados de $10 \times 10 \mathrm{~cm}$, nas duas estações (Brachidontes e Cirripedia) as quais foram acompanhadas quinzenalmente, durante 6 meses no período de agosto de 2004 a janeiro de 2005, no horário de baixa-mar (DHN, 2004; 2005).

Nas estações foram delimitados transectos no sentido paralelo á linha de praia de 2 metros de comprimento equivalente a 20 quadrados alinhados horizontalmente. Dos quais foram sorteados 9 quadrados sendo considerados 3 de recrutamento e 3 de sucessão e 3 de controle. Os quadrados de sucessão e recrutamento foram delimitados e marcados com cimento hidráulico, uma massa á prova de água.

Na primeira coleta, realizada em agosto de 2004, foi feita a primeira contagem dos organismos para avaliar a densidade dos mesmos usando como unidade amostral os 9 quadrados sorteados. Posteriormente foram raspados 3 quadrados de recrutamento e 3 quadrados de sucessão. As amostras coletadas foram acondicionadas em frascos com álcool $70 \%$ e levadas ao laboratório de Biologia Animal do Departamento de Biologia da Universidade Pernambuco para identificação e mensuração. Nas 10 coletas seguintes realizadas em intervalos médios de 15 dias, os quadrados de recrutamento continuaram a serem raspados, enquanto os de sucessão e controle eram estudados em campo sem perturbações adicionais. Os quadrados de controle eram escolhidos aleatoriamente entre os 14 quadrados restantes.

As médias e desvios plotados nas Figuras 1, 2, 3 e 4 na primeira coleta representam a valores populacionais observados antes da perturbação artificial, enquanto os dados seguintes fornecem informação pós-perturbação. Utilizamos para a análise dos dados a ANOVA paramétrica para os quadrados permanentes (aleatórios) e ANOVA não paramétrica para os quadrados de sucessão e recrutamento.

Amostras de plâncton de duas poças ao redor das estações foram coletadas, e levadas ao Laboratório de Zooplâncton do Departamento de Oceanografia da UFPE para análise da comunidade zooplanctônica. Uma poça apresentava maior distância em relação á área de arrebentação, tendo água parada e menos turva. Tais amostras foram tomadas com auxilio de um balde graduado (capacidade para 10 litros), sendo 30 litros de água de cada poça filtrados em malha de rede de $64 \mu \mathrm{m}$, colada em uma extremidade de filtro cilíndrico de PVC, com $20 \mathrm{~cm}$ de altura por $15 \mathrm{~cm}$ de diâmetro. Foi avaliada nesta etapa apenas a freqüência de ocorrência dos grupos de organismos.

\section{RESULTADOS E DISCUSSÃO}

O tamanho das aberturas utilizadas em nosso experimento estava dentro da escala observada para aberturas naturais. A composição de espécies não diferiu nas diferentes estações. A fauna obtida nas amostras pertence a diferentes grupos, como Crustacea (Chthamalus sp. e Balanus sp.), Mollusca (Brachidontes sp., Collisella subrugosa, Fissurella clenchi, Isopoda, Pinetada imbricata, Tricolia affinis, Littorina ziczac), Annelida (Polichaeta Nereis sp.), Nematoda.

Na primeira coleta observamos o grande número de Brachidontes (Figura 4), porém a variação da comunidade não mostrou muitos resultados estatisticamente significativos entre as datas de coleta sugerindo uma distribuição não uniforme das espécies dominantes, nos quadrados de controle (Figuras 1, 2, 3 e 4). Nos quadrados de sucessão, que foram raspados apenas na primeira e última coleta, o número de indivíduos de bivalves foi baixo, chegando a zero em algumas datas, mostrando que seu recrutamento não foi significativo no período do estudo (Figuras 1, 2, 3). Verificamos que somente para quadrados de sucessão de Cirripedia na estação 2 (dominada por cracas) houve recrutamento significativo, apresentando valores com diferenças estatísticas significativa entre algumas datas de coleta (Figura 1 e 2), com uma recuperação razoável dos valores de densidade quando comparados com a população controle. A diferença estatística significativa $(p<0,05)$ só foi observada no experimento de sucessão de cracas na faixa de Cirripedia (Figura 1). A população mostrou uma tendência de aumento com a progressão do experimento e a variação significativa foi observada entre as seguintes datas de coleta: 2 e 9, 10, 11; 4 e 
10,$11 ; 7$ e 10,11. Um pico de recrutamento de cracas foi observado (Figura 1), sugerindo que pulsos de eventos de recrutamento podem estar relacionados com a manutenção e recuperação das populações de invertebrados, a variabilidade temporal observada nos eventos de recrutamento têm sido relacionados com fatores ambientais físicos como temperatura da água e circulação (SKINNER, 2003, NAVARRETE et al., 2002).

A população de bivalves não mostrou recuperação significativa da comunidade durante o tempo do experimento, porém tempos similares de experimentos (alguns meses) mostram recuperação das populações de Brachidontes após perturbações artificiais (TANAKA, 2005) em outras regiões entre-marés, contudo o mesmo autor sugere que alguns meses podem não ser suficiente para observar o processo completo de sucessão de invertebrados. Como a recuperação relativa das populações de Cirripedia foi observada durante o período de estudo os dados de campo e da literatura sobre a dinâmica populacional destes organismos foram utilizados no desenvolvimento de simulações computacionais (Figura 5).

Podemos notar que nas demarcações dos quadrados, feitas com cimento hidráulico, havia presença de organismos jovens de Cirripedia, esse número chegou a 114 organismos na sexta coleta. Nas amostras planctônicas, retiradas das poças, mostraram que Balanus sp. foi encontrado em forma de náuplios e cipris, sugerindo que a disponibilidade de larvas pode contribuir para o recutamento e recuperação das populações de invertebrados, porém o número de Chthamalus sp. em relação ao de Balanus sp. na primeira coleta foi maior em todos os quadrados.

A visualização em campo, devido ao tamanho muito pequeno dos organismos e a grande quantidade de sedimento que cobria os quadrados, foi bastante dificultada, porém de um modo geral foi observada a recuperação relativa da comunidade da populações de cracas na faixa de Cirripedia. Apesar do recrutamento ter sido variável com eventuais taxas mais altas em certas datas de coleta (Figura 2). Houve uma acentuada presença de algas nas amostras, organismos pioneiros de sucessão (algas). A presença de Collisella subrugosa e Fissurella clenchi foi observada, trabalhos mostram que em aberturas pequenas como as que foram feitas em nosso trabalho, estes herbívoros gastrópodes apropriam-se mais rapidamente do espaço aberto pela migração lateral atraídos pelas algas.

Muitos poliquetas (Nereis sp.) estavam presentes nas amostras na primeira coleta, quando os quadrados estavam com cobertura total. Nas coletas seguintes sua presença não foi notada. SKINNER \& COUTINHO (2005) mostram que o recrutamento de larvas de Cirripedia foi maior em blocos de substrato rugoso o que pode afeta a distribuição local dos organismos, porém os experimentos aqui descritos sugerem que a sedimentação do recife e processos de eutroficação, podem prejudicar a recuperação das populações de invertebrados.

Com ajuda do paquímetro medimos o tamanho dos organismos levados a laboratório, na primeira raspagem para Brachidontes a média foi de $1,0 \mathrm{~cm}$ e para Cirripedia $1,5 \mathrm{~cm}$ de altura por $0,75 \mathrm{~cm}$ de largura na base em média. Nas raspagens seguintes os organismos que recrutaram tiveram tamanhos muito pequenos, para Brachidontes uma média de 0,2 $\mathrm{cm}$ e para Cirripedia 0,5 cm de altura e 0,1 de base em média.

A avaliação planctônica revelou uma baixa densidade de organismos nas poças intertidais, se comparada com uma amostra realizada em estuário, isto provavelmente se deve pelo volume de água de cada poça estudada (no caso de Piedade estima-se que a maior poça estudada tenha aproximadamente 100 litros de água).

De modo geral, todas as espécies encontradas nas amostras de plâncton são típicas de áreas costeiras de Pernambuco, indicadoras de plâncton costeiro (NEUMANN- LEITÃO et al., 1998; PORTO NETO, 2003). Os mais abundantes foram os Foraminifera, seguidos por Euterpina acutifrons, Oithona hebes e Gastropoda. Em cerca de 100 litros de água foram registrados cerca de 50 Foraminifera, de varias espécies, principalmente da família Trochaminidae, fato também observado por PORTO NETO (2003) estudando o plâncton recifal de Tamandaré. Os demais taxa não ultrapassam mais de 5-7 org./100 L, exceto náuplios de Copepoda, sempre abundantes, que atingiram até 25 org./100 L. Gastropoda e Bivalvia também tiveram destaque nas amostras, e estes grupos também são amplamente encontrados no bentos da praia de Piedade. Dentre os Copepodas, destacam-se Oithona 
ALMEIDA, et al. Interação entre processos de colonização e sucessão interação entre processos de colonização e sucessão ecológica de comunidades bioincrustantes na praia de Piedade.

hebes e Parvocalanus crassirostris, muito comuns em áreas costeiras do litoral de Pernambuco (NEUMANN- LEITÃO et al., 1998; PORTO NETO, 2003).

Houve uma notada influencia do bentos nas duas poças estudas, por Foraminifera, Amphipoda, e Copepoda (este ultimo grupo representado principalmente por Harpacticoida, como Trigiopus sp.). Essa influência do bentos se dá principalmente pela baixa profundidade de cada poça (poucos centímetros a meio metro no máximo) e pela constante movimentação de água (ondas que entram nas poças, pisoteio, ventos, etc). Por esta razão também foi encontrado muito material particulado nas amostras (areia fina, sedimento bioclástico, e restos de conchas e macroalgas).

Não foi calculada a densidade de todos os organismos, sendo feito apenas um qualitativo das amostras, devido á impossibilidade de se estimar o volume exato das poças de maré, sendo calculada apenas a freqüência de ocorrência dos grupos mais importantes (Tabela 1). A baixa diversidade encontrada nas amostras de plâncton intertidal serve de alerta para problemas ambientais na região costeira de Piedade.

Os resultados obtidos em nosso estudo de colonização serviram de base para a elaboração de um modelo com o software Stella ${ }^{T M}$ que assume o recrutamento como função de entrada de novos indivíduos da população a cada passo de tempo e simula bem em ordem de grandeza (centenas de indivíduos em $100 \mathrm{~cm}^{2}$ ) do quantitativo dos invertebrados encontrados nos quadrados amostrados no campo (Figuras 1, 2, 3, 4 e 5). Leva em consideração a equações: $\operatorname{Cracas}(t)=\operatorname{Cracas}(t-d t)+($ Crescimento - Mortalidade $) * d t(1)$, Crescimento $=$ Cracas*Recrutamento* $(1-($ Cracas $/ K))$ (2), Mortalidade $=$ Taxa_de_Mortalidade*Cracas (3). O recrutamento está representado na Figura 5 e representa a ordem de grandeza de recrutas observados no campo em algumas datas de coleta (variando entre 0 e duas dezenas de indivíduos por $100 \mathrm{~cm}^{2}$ ) por passo médio de tempo da simulação (dias), os valores também estão de acordo com a ordem de grandeza registrada com a literatura sobre recrutamento de invertebrados de substrato consolidado (SKINNER \& COUTINHO, 2002, SKINNER, 2003, NAVARRETE et al., 2002). Os parâmetros do modelo foram taxa de mortalidade de 5 indivíduos por passo de tempo e capacidade de suporte $(K)$ de 1000 indivíduos, o experimento teve uma condição inicial de 5 cracas por $100 \mathrm{~cm}^{2}$.

Trabalhos mostram que a área de estudo é bastante impactada, podendo ser considerada como local bastante modificado. A poluição orgânica favorece o aparecimento de espécies oportunistas e de ciclo de vida curto que dominam a área reduzindo a diversidade de toda a biota marinha (SOUSA \& COCENTINO, 2004) como as macroalgas verdes. Os principais impactos antrópicos observados foram devido à alta concentração urbana que tem gerado um acréscimo de matéria orgânica na área, sendo necessário medidas que visem minimizar este impacto, através da implementação de programas de educação ambiental para conscientizar a população da importância da preservação dos ambientes recifais.

Os dados das amostras de plâncton mostraram que a região de estudo possui um bom potencial de recrutamento para Cirripedia, porém em praticamente toda extensão das 2 estações, houve apenas um quadrado na área de Cirripedia que teve uma taxa significativa de recrutamento, mas a recuperação ao nível de abundância pré-impacto não foi observada. O processo de sedimentação que o recife está sofrendo, pode ter sido também, um dos agentes que dificultaram o desenvolvimento e a sucessão das aderências orgânicas. Chegamos à conclusão que a grande agressão física sofrida pela região não está permitindo que sua fauna se recupere e que o tempo de estudo deve ser aumentado para acompanhar a real capacidade de recuperação da comunidade em questão.

\section{REFERÊNCIAS BIBLIOGRÁFICAS}

APOLINÁRIO, M., Temporal variations in community structure in and around intertidal barnacle Chthamalus challengeri Hoek patches on a plebby shore in Japan. Revista Brasileira de Biologia, v. v. 59, n. 1, p. 4-53, 1999.

CAVALCANTI, L. B. \& KEMPF, M. Estudo da Plataforma Continental na Área do Recife (Brasil). II. Metereologia e Hidrologia. Trabalhos Oceanográficos da UFPE, v. 9-11, p. 149$158,1967 / 69$. 
COUTINHO, R., Bentos de costões rochosos. In: Pereira R. C. \& Soares-Gomes (Organizadores) Biologia Marinha. Rio de Janeiro: Editora Interciência, 2002, p. 147-158.

CHASILEW, S., Colonização, recrutamento e sucessão ecológica de organismos bentônicos sob diferentes densidades de cirrípedes. Niterói, 50 f., Dissertação de Mestrado, Universidade Fluminense (UFF), 1998.

GROBLER, N. J.; VAN AS, J. G.; OLIVIER, P. A.S. Description of the previously unknown male of Caligus mortis Kensley, 1970 (Copepoda: Caligidae), parasite of intertidal fish from South Africa. Folia Parasitológica, v. 49, n. 2, p. 131-136, 2002.

JOHNSON, M. P. Metapopulation dynamics of Tigriopus brevicornis (Harpacticoida) in intertidal rock pools. Marine Ecology Progress Series, v. 211, p. 215-224, 2001.

KIM, J.H.; DeWREEDE, R.E.. Effects of size and season of disturbance on algal patch recovery in a rocky intertidal community. Marine Ecology Progress Series, v. 133, p. 217228,1996

LEWIN, S. A., Supply-side ecology. Science, v. 234, p. 25-27, 1986.

LUBCHENCO, J. \& MENGE, B. A., Community development and persistence in a low rocky intertidal zone. Ecological Monographs, v. 48, p. 67-94, 1978.

ODUM, E. P., Ecologia. Rio de Janeiro: Editora Guanabara, 1988.

NAVARRETE, S. A., BROITMAN, B., WIETERS, E. A., FINKE, G. R., VENEGAS, R. M. \& SOTOMAYOR, A., Recruitment of intertidal invertebrates in the southeast Pacific:

Interannual variability and the 1997-1998 El Ninõ. Limnology and Oceanography, v. 47, p. 791-802, 2002.

NEUMANN-LEITÃO, S.; GUSMÃO, L.M.O.; NASCIMENTO-VIEIRA, D.A.; PORTO NETO, F. de F.; MOURA, M.C.O.; SILVA, A.P. Biodiversidade e Produtividade do Zooplâncton Estuarino de Pernambuco (Brasil). In: $4^{\circ}$ Congresso de Ecologia do Brasil, 1998, BELÉM. Abstract, 1998.

PINTO-COELHO, R. M., Fundamentos em Ecologia - Porto Alegre: Artes Médicas Sul, 2000.

PORTO NETO, F. de F., Zooplankton as bioindicator of environmental quality in the Tamandaré reef system (Pernambuco, Brazil): anthropogenic influences and interaction with mangroves. Bremen, 131 f., Ph. D. Dissertation, ZMT Bremen, 2003.

SAUER-MACHADO, K.R.S., Chapman, A.R.O. \& Coutinho, R. Patch structure in a tropical rocky shore community in Brazil: a mosaic of sucessional states? Ophelia, v. 35, p. 187195, 1992.

SOUSA, G.S. \& COCENTINO, A. L. M., Macroalgas como indicadoras da qualidade ambiental. Tropical Oceanography, v. 32, p. 1-22, 2004.

SKINNER, L. F., Processos determinantes da estrutura populacional dos crustácea: cirripedia Chthamalus bisinuatus e Tetraclita stalactifera na área de influência da ressurgência do Cabo Frio, RJ, Brasil. Rio de Janeiro, 105 f. Tese de doutorado, Universidade do Estado do Rio de Janeiro (UERJ), 2003.

SKINNER, L. F.; COUTINHO, R. Preliminary results on settlement of barnacles Tetraclita stalactifera and Chthamalus bisinuatus on a Brazilian tropical rocky shore under upwelling conditions. International Journal of Invertebrate Reproduction and Development, v. 41, n. 1-3, p. 151-156, 2002.

SKINNER, L. F. \& COUTINHO, R. Effect of microhabitat distribution and substrate roughness on barnacle Tetraclita stalactifera (Lamark, 1818) settlement. Brazilian Archives of Biology and Technology, v. 48, p. 109-113, 2005.

TANAKA, M. O. \& MAGALHÃES, C. A., Edge effects and succession dynamics in Brachidontes mussel beds. Marine Ecology Progress Series, v. 237, p. 151-158, 2002.

TANAKA, M. O. Recolonization of experimental gaps by the mussels Brachidontes darwinianus and B. solisianus in a Subtropical Rocky Shore. Brazilian Archives of Biology and Technology, v. 48, p. 115-119, 2005. 